\title{
Potencial uso da casca de café como constituinte de substrato para produção de mudas de espécies florestais
}

\author{
Potential use of coffee husk as a substrate constituent for the production of \\ forest species seedlings
}

\author{
Oclizio Medeiros das Chagas SilvaI, Marileydy Martínez Hernández ${ }^{\mathrm{II}}$, \\ Geisislaine do Carmo Reis Araújo ${ }^{\mathrm{III}}$, Fernanda Leite Cunha ${ }^{\mathrm{III}}$, \\ Djavan Valentim da Paixão Evangelista ${ }^{I V}$, Paulo Sérgio dos Santos Leles ${ }^{\mathrm{V}}$, \\ Lucas Amaral de Melo ${ }^{\mathrm{VI}}$
}

\begin{abstract}
Resumo
Na produção de mudas de espécies florestais há vários fatores que influenciam no seu crescimento. Dentre estes, um dos mais importantes é a composição do substrato. Nesse sentido, é essencial a seleção de bons constituintes que atendam a essa demanda. O objetivo deste trabalho foi avaliar o crescimento inicial de mudas de Eucalyptus urophylla, Peltophorum dubium e Eremanthus erythropappus, produzidas em diferentes formulações de substratos. Para cada espécie, foi instalado e conduzido um experimento, utilizando-se nas formulações os constituintes casca de arroz compostada, casca de café compostada, fibra de coco e substrato comercial. Os experimentos foram instalados em delineamento inteiramente casualizado (DIC), constituídos por oito tratamentos, cinco repetições e 20 mudas por parcela. Para avaliação da qualidade das mudas, aos 120 dias foram mensuradas as variáveis altura, diâmetro do coleto, peso de matéria seca da parte aérea, peso de matéria seca do sistema radicular e índice de qualidade de Dickson. Como análises qualitativas, foram avaliadas a facilidade de retirada das mudas do tubete e a agregação das raízes ao substrato. Os resultados encontrados após a avaliação atestaram que as mudas produzidas nos tratamentos formulados com casca de café compostada apresentaram valores superiores para a maioria das variáveis analisadas, quando comparado com as mudas produzidas nos tratamentos formulados com a casca de arroz e na testemunha. Para as três espécies, indica-se a utilização da proporção de $40 \%$ de casca de café compostada com $40 \%$ de substrato comercial e $20 \%$ de fibra de coco para composição do substrato.
\end{abstract}

Palavras-chave: Crescimento inicial; Composto orgânico; Materiais alternativos; Propagação de plantas

\footnotetext{
Engenheiro Florestal, Me., Doutorando em Ciências Ambientais e Florestais, Universidade Federal Rural do Rio de Janeiro, Instituto de Florestas, Departamento de Silvicultura, BR 465, km 07, CEP 23890-000, Seropédica (RJ), Brasil. omflorestal@hotmail.com (ORCID: 0000-0003-3099-8919)

Engenheira em Inovação Agrícola Sustentável, Ma., Doutoranda em Engenharia Florestal, Universidade Federal de Lavras, Laboratório de Silvicultura, Campus Universitário, CEP 37200-000, Lavras (MG), Brasil. deydy 17@ hotmail.com (ORCID: 0000-0003-0968-6849)

III Engenheira Florestal, Ma., Universidade Federal de Lavras, Laboratório de Silvicultura, Campus Universitário, CEP 37200-000, Lavras (MG), Brasil. geisecraraujo@gmail.com (ORCID: 0000-0001-9324-698X) / fernandaleitecunha@gmail.com (ORCID: 0000-0001-7707-0910)

Iv Engenheiro Agrônomo, Mestrando em Biotecnologia Vegetal, Laboratório de Biologia Molecular, Universidade Federal de Lavras, Campus Universitário, CEP 37200-000, Lavras (MG), Brasil. dj.avanpaixao@hotmail.com (ORCID: 000-0003-0785-6269)

Engenheiro Florestal, Dr., Professor Titular do Instituto de Florestas, Universidade Federal Rural do Rio de Janeiro, Instituto de Florestas, Departamento de Silvicultura, BR 465, km 7, CEP 23890-000, Seropédica (RJ), Brasil. pleles@ufrrj.br (ORCID: 0000-0002-8393-6095)

vı Engenheiro Florestal, Dr., Professor adjunto do Departamento de Ciências Florestais, Universidade Federal de Lavras, Laboratório de Silvicultura, Campus Universitário, CEP 37200-000, Lavras (MG), Brasil. lucas.amaral@dcf.ufla.br (ORCID: 0000-0001-5219-9179)
} 


\begin{abstract}
In the production of forest species seedlings there are several factors that influence their growth. Among these, one of the most important is the composition of the substrate. In this sense, it is essential to select good constituents that meet this demand. The objective of this work was to evaluate the initial growth of seedlings of Eucalyptus urophylla, Peltophorum dubium and Eremanthus erythropappus, produced in different substrate formulations. For each species, an experiment was installed and conducted, using composted rice husk, composted coffee husk, coconut fiber and commercial substrate in the formulations. The experiments were installed in a completely randomized design (DIC), consisting of eight treatments, five replications and 20 seedlings per plot. To evaluate the quality of seedlings, at 120 days the variables height, stem diameter, dry matter weight of the aerial part, dry matter weight of the root system and Dickson's quality index were measured. As qualitative analyzes, the ease of removing seedlings from the tube and the aggregation of roots to the substrate were evaluated. The results found after the evaluation attested that the seedlings produced in the treatments formulated with composted coffee husk presented higher values for most of the analyzed variables, when compared with the seedlings produced in the treatments formulated with the rice husk and in the control. For the three species, the use of the proportion of $40 \%$ of coffee husk composted with $40 \%$ of commercial substrate and $20 \%$ of coconut fiber for the composition of the substrate is indicated.
\end{abstract}

Keywords: Initial growth; Organic compound; Alternative materials; Plant propagation

\title{
Introdução
}

O sucesso obtido na produção de mudas florestais está diretamente ligado ao tipo de substrato utilizado, sendo que mudas de qualidade são de fundamental importância para a formação de povoamentos florestais. Para atender a tais requisitos, a seleção de constituintes para formulações de substratos deve ser levada em consideração, pois a sobrevivência e o crescimento da muda em campo estão relacionados com sua formação inicial (DA ROS et al., 2015; MELO et al., 2018).

O substrato tem a função de sustentar e fornecer condições adequadas para a muda, contribuindo para seu crescimento inicial, garantindo, consequentemente, maior sobrevivência em campo e resistência a estresses ambientais (KRATZ et al., 2016), influenciando diretamente na qualidade final da floresta. Nesse sentido, pesquisas têm sido realizadas com o intuito de selecionar constituintes capazes de melhorar a qualidade da muda (KRATZ et al., 2013b; FARIA et al., 2016; ABREU et al., 2017), entre vários outros objetivos.

Dentre as características desejáveis na seleção de constituintes para substratos, destacamse o custo-benefício, a disponibilidade na região, a esterilidade biológica e o fácil manuseio (KRATZ et al., 2013a; ABREU et al., 2017). Deve-se atentar também para as características físicas, como densidade, porosidade, macro e microporosidade, e químicas, como $\mathrm{pH}$ e condutividade elétrica, que influenciam significativamente no crescimento da muda (WENDLING et al., 2007; KRAUSE et al., 2017). Recomenda-se a mistura de materiais diversos, com o intuito de formular um substrato com condições apropriadas para favorecer o crescimento da muda (GERVASIO et al., 2016; SOUSA et al., 2016).

Na produção de mudas, os constituintes mais comumente utilizados nas formulações dos substratos são: casca de arroz carbonizada, vermiculita, areia, moinha de carvão, turfa, serragem, esterco bovino, biossólido e diversas misturas desses constituintes (SILVA et al., 2012). Constituintes alternativos, como fibra de coco e palha de café, este último, ainda com poucos estudos, têm apresentado boas características físicas (CALDEIRA et al., 2013; KRATZ et al., 2016) e são opções viáveis para promover alterações na qualidade dos substratos.

$\mathrm{O}$ uso de resíduos encontrados na região, em fontes de fácil disponibilidade, pode contribuir para a redução do custo do substrato (MELO et al., 2014; ABREU et al., 2017), e, por conseguinte, influenciar no preço final da muda. Dentre os resíduos, destaca-se a casca de café, que pode ser obtida em vários estados brasileiros que cultivam a cafeicultura. Entre as 
regiões produtoras, o estado de Minas Gerais se destaca como grande produtor de café, o qual teve produção estimada em 30,7 milhões de sacas, na safra de 2018 , correspondendo a $53 \%$ da produção nacional (CONAB, 2018).

Entre as regiões produtoras do estado de Minas Gerais, a produção da Região Sul foi estimada em aproximadamente 16 milhões de sacas, gerando ampla quantidade de resíduos no final do seu processamento. $O$ rendimento da palha de café no processo de beneficiamento do fruto é cerca de 1:1, gerando aproximadamente 500 mil toneladas de resíduo (CONAB, 2018), originando um material com possibilidade de ser aplicado como constituinte para substrato, devido a sua fácil disponibilidade e seu baixo custo de aquisição, podendo este ser empregado na produção de mudas na região.

A casca de café é utilizada, por alguns produtores, como adubação orgânica nas entrelinhas das lavouras de café e, em muitos casos, este resíduo é simplesmente descartado na natureza. Com o intuito de aproveitar esse material de forma sustentável, sugere-se a sua utilização como constituinte alternativo para a composição de substrato, o qual poderá gerar uma série de benefícios à natureza, proporcionando redução do volume de resíduos, além de amortizar o custo final do produtor, no processo de produção de mudas.

Devido à importância do substrato na produção de mudas e à procura por materiais alternativos, objetivou-se, neste trabalho, avaliar o uso de constituintes à base de casca de arroz compostada, casca de café compostada, fibra de coco e substrato comercial, na produção de mudas de três espécies florestais, e também relacionar as propriedades físico-químicas dos substratos formulados, em relação à muda formada.

\section{Material e métodos}

\section{Localização e caracterização da área de estudo}

Para fins de avaliação do potencial dos constituintes do substrato na produção de mudas, foi instalado um teste em um viveiro florestal localizado no Sul de Minas Gerais, no período de agosto a dezembro de 2018. Para o estudo, foram utilizadas três espécies arbóreas, sendo elas: Eucalyptus urophylla (S. T. Blake), Peltophorum dubium (Spreng.) e Eremanthus erythropappus (DC.) MacLeish.

Conforme a classificação climática de Köppen, o clima da região é Cwa, temperado chuvoso (mesotérmico) com inverno seco e verão chuvoso, subtropical, sendo a temperatura do mês mais quente maior que $22^{\circ} \mathrm{C}$, com temperatura média de $19,9^{\circ} \mathrm{C}$, apresentando pluviosidade média anual de $1486 \mathrm{~mm}$ (ALVARES et al., 2013).

\section{Obtenção e preparo dos constituintes para formulação dos substratos}

Os substratos empregados na produção das mudas foram formulados com quatro tipos de constituintes: casca de café compostada, casca de arroz compostada, fibra de coco e substrato comercial Maxfértil. Como testemunha foi formulada a proporção de $20 \%$ de fibra de coco, de textura grosseira, e $80 \%$ de substrato comercial Maxfértil, sendo este composto por casca de pinus, fosfato natural, casca de arroz carbonizada e vermiculita.

A casca de arroz foi adquirida de uma empresa de beneficiamento de arroz localizada no Centro-Oeste de Minas Gerais, ao passo que a casca de café utilizada foi obtida por meio de doação de cafeicultores rurais nas proximidades do local de produção das mudas. O esterco bovino foi cedido por um produtor rural de uma propriedade próxima ao viveiro. Este foi coletado em currais de vacas leiteiras, previamente selecionado e curtido por 30 dias. A fibra de coco, de textura grosseira, e o substrato comercial foram obtidos em loja de insumos agropecuários. 
Para produção do composto orgânico, foi escolhido um local aberto nas dependências do viveiro. Para montagem da pilha de compostagem, utilizou-se $500 \mathrm{~L}$ de esterco bovino, e a mesma proporção, com casca de café in natura. A técnica de compostagem foi baseada em um processo espontâneo de decomposição de materiais orgânicos que passaram por processo de estabilização biológica, por período de 120 dias, em local aberto e, posteriormente, peneirados em malha de aço de $3 \mathrm{~mm}$ para completa homogeneização das partículas. O mesmo procedimento foi aplicado para a compostagem da casca de arroz.

Posteriormente, foram quantificadas as proporções de cada constituinte e realizada a mistura com o uso de uma betoneira, até completa homogeneização. Para favorecer o crescimento das mudas na fase inicial, durante a mistura dos constituintes, foi adicionado adubo de liberação lenta (Osmocote), na proporção de $4 \mathrm{~kg} \mathrm{~m}^{-3}$ de substrato.

\section{Delineamento experimental}

Para cada espécie, foi conduzido um experimento, cada um foi instalado em delineamento inteiramente casualizado (DIC), constituído de oito tratamentos, com cinco repetições e 20 plantas por parcela. As proporções dos tratamentos estão dispostas na Tabela 1.

Tabela 1 - Proporções volumétricas dos constituintes (\%) utilizados na formulação dos substratos para a produção de mudas de Eucalyptus urophylla, Peltophorum dubium e Eremanthus erythropappus

Table 1 - Volumetric proportions of the constituents (\%) used in the formulation of the substrates for the production of Eucalyptus urophylla, Peltophorum dubium and Eremanthus erythropappus seedlings

\begin{tabular}{ccccc}
\hline Tratamentos & FC & SC & COA & COC \\
\hline T1 & 20 & 20 & 60 & - \\
T2 & 20 & 40 & 40 & - \\
T3 & 20 & 60 & 20 & - \\
T4 & 20 & 20 & 30 & 30 \\
T5 & 20 & 40 & - & 60 \\
T6 & 20 & 40 & - & 40 \\
T7 & 20 & 60 & - & 20 \\
T8 & 20 & 80 & - & - \\
\hline
\end{tabular}

Fonte: Autores (2019)

Em que: FC - Fibra de coco; SC - Substrato comercial; COA - Composto orgânico da casca de arroz; COC Composto orgânico da casca de café.

\section{Análises químicas e físicas dos substratos}

Com a finalidade de realizar a caracterização física e química, amostras dos substratos formulados foram encaminhadas ao Laboratório de Substratos do Departamento de Horticultura e Silvicultura da Universidade Federal do Rio Grande do Sul (UFRGS). Na caracterização química dos substratos, foram avaliados o potencial hidrogeniônico $(\mathrm{pH})$, determinado em água, diluição 1:5 (v/v) e condutividade elétrica (CE), obtida em solução 1:5 (v/v), de acordo com metodologia descrita na Instrução Normativa nㅜㄹ, de 21 de maio 2007 (MAPA, 2007).

Para as análises físicas, foram avaliados a densidade úmida (DU), a densidade seca (DS), 
a porosidade total (PT), o espaço de aeração (EA) e a capacidade de retenção de água (CRA), sob sucção de $50 \mathrm{~cm}$ de coluna de água, determinado em base volumétrica. Para melhor compreensão e confiabilidade dos dados, foram realizadas três replicatas por amostras,tanto para análises químicas,quanto para as físicas.

\section{Processo de produção de mudas}

Para a produção das mudas, as sementes das espécies foram coletadas na Região Sul de Minas Gerais. Após a formulação dos substratos, estes foram acondicionados em tubetes com capacidade de $55 \mathrm{~cm}^{3}$ para o Eucalyptus urophylla e de $110 \mathrm{~cm}^{3}$ para o Peltophorum dubium e Eremanthus erythropappus, os quais foram alocados em bandejas de polipropileno, apoiadas em canteiros suspensos a aproximadamente $80 \mathrm{~cm}$ do solo.

A semeadura foi realizada diretamente nos tubetes, colocando entre três a quatro sementes por recipiente. Após 15 dias da emergência das plântulas, foi realizado o raleio, deixando uma plântula por recipiente, escolhendo a mais vigorosa e mais centralizada. A irrigação das mudas foi feita por sistema de microaspersão, três vezes ao dia, em lâmina fina de água, com duração de sete minutos cada, com consumo médio diário de $15 \mathrm{~mL} /$ tubete. Aos 60 dias, foi realizada a alternagem das mudas, com o objetivo de reduzir a competição por luz e aumentar o espaçamento, contribuindo para o melhor crescimento.

Durante o processo de produção das mudas, foram realizados tratos culturais, com a retirada de plantas daninhas e musgos dos recipientes para facilitar o crescimento das mudas. Com 45 dias após a emergência, em intervalos de 15 dias,eram aplicados cloreto de potássio (KCL) e monoamônio fosfato (MAP) como adubação de cobertura, na proporção de 100 e 1000 g, respectivamente, diluídos em $100 \mathrm{~L}$ de água, aplicados com o auxílio de regador. Para evitar a ocorrência de doença fúngica, que pode ocasionar tombamento nas mudas de Eremanthus erythropappus, foi realizadoo tratamento com aplicação de pencicurom (feniluréia), na dosagem de $3 \mathrm{ml} \mathrm{L}^{-1}$, aplicando a solução sobre as mudas com auxílio de regador.

\section{Caracterização morfológica e qualitativa das mudas produzidas}

Com a finalidade de avaliação de qualidade, aos 120 dias após semeadura, com o intuito de evitar o efeito de borda, foram mensuradas as mudas centrais das parcelas, avaliando a altura da parte aérea $(\mathrm{H})$ e o diâmetro do coleto (DC). Para a obtenção do peso da matéria seca da parte aérea (MSPA) e peso da matéria seca do sistema radicular (MSR), foram escolhidas quatro mudas por parcela, aquelas que mais se aproximaram da média de altura e diâmetro do coleto.

Posteriormente, foram separadas a parte aérea do sistema radicular, sendo esta última lavada em água corrente para retirada do substrato. Em seguida, foram identificadas e embaladas em sacos de papel, as quais foram acondicionadas em estufa, com circulação forçada de ar e temperatura de $65^{\circ} \mathrm{C}$ por $72 \mathrm{~h}$, sendo posteriormente pesadas em balança de precisão. De posse destes dados, foram calculados o peso da matéria seca total (MST) e o índice de qualidade de Dickson (IQD), sendo este último obtido por meio da fórmula de Dickson, Equação 1, (DICKSON et al., 1960).

$$
I Q D=\frac{M S T(g)}{H(c m) / D C(m m)+M S P A(g) / M S R(g)}
$$

onde: MST é o peso da matéria seca total; $H$ é a altura; $D C$ é o diâmetro do coleto; MSPA é o peso matéria seca da parte aérea; MSR é o peso da matéria seca radicular.

Para avaliação da qualidade do torrão formado, foi feita a avaliação de facilidade de retirada das mudas do tubete (FRT) e agregação das raízes ao substrato (AGR), adaptando à metodologia descrita por Wendling et al. (2007). Para este procedimento, também foram selecionadas quatro 
mudas por parcela, conforme etapa anterior. Para avaliação da FRT, foram atribuídas notas de um a dez, sendo um a dificuldade máxima e dez a facilidade máxima de retirada das mudas do recipiente, após três batidas na parte superior do tubete.

Quanto à AGR, após as mudas serem retiradas dos tubetes, elas foram soltas em queda livre a cerca de um metro do solo. Ao torrão esboroado foi atribuída nota de um a dez, sendo um, para a muda totalmente esboroada e dez, para o torrão íntegro.

\section{Análises estatísticas}

Os dados gerais obtidos foram submetidos à análise de normalidade dos erros pelo teste de Shapiro-Wilk a 5\% de probabilidade de erro, utilizando o software estatístico R (R CORE TEAM, 2016). Posteriormente, foi empregada a análise de variância (ANOVA), e uma vez verificada diferença significativa, foi realizada a comparação de médias pelo teste Tukey, em nível de $5 \%$ de probabilidade de erro, utilizando o software Sisvar 5.6 (FERREIRA, 2014).

Para melhor descrição da influência das diferentes formulações dos substratos, na qualidade das mudas produzidas, foi realizada a análise de componentes principais (PCA), por meio da qual foi possível agrupar os tratamentos conforme seu desempenho. A PCA foi realizada baseada na matriz de correlação, utilizando o software R OriginLab Versão 8.6 (LI et al., 2018).

\section{Resultados e discussão}

Os dados referentes às análises químicas e físicas dos substratos formulados estão dispostos na Tabela 2. $\mathrm{Na}$ avaliação do $\mathrm{pH}$, observou-se que os valores dos tratamentos variaram de 4,46 a 5,67. De acordo com Gonçalves e Poggiani (1996), a faixa de pH ideal para a maioria das culturas florestais situa-se na faixa de 5,5 a 6,5. Porém, conforme Kratz et al. (2013a), quando são utilizados substratos orgânicos e quando não há adição de solo em sua composição, recomendase trabalhar com pH em intervalo de 4,4 a 6,2, corroborando, portanto, com os valores reportados neste estudo.

Conforme Valeri e Corradini (2000), quando o pH está abaixo de 5,0, há problemas na absorção de nitrogênio, cálcio, magnésio, potássio e boro, ao passo que, quando ultrapassa valores de 6,5, pode ocorrer sintomas de deficiência de fósforo, ferro, zinco e cobre. Dessa forma, as propriedades químicas dos substratos influenciam na disponibilidadede macro e micronutrientes, e, por consequência, podem afetar o crescimento das mudas, pois a absorção de nutrientes é afetada.

$\mathrm{Na}$ avaliação da condutividade elétrica (CE), houve baixa variação entre as formulações dos substratos. Contudo, o maior valor $\left(1,65 \mathrm{mS} \mathrm{cm}^{-1}\right)$ foi observado no tratamento $\mathrm{T} 5$, composto por $20 \%$ de fibra de coco, $20 \%$ de substrato comercial e $60 \%$ de casca de café compostada. Provavelmente essevalor está associado a alta porcentagem de casca de café presente na formulação e os compostos existentes nesse material podem ter ocasionado o aumento da concentração de sais no substrato. Valores de CE acima de $3,0 \mathrm{mS} \mathrm{cm}^{-1}$ são considerados excessivos e podem danificar as raízes das plantas e os pelos radiculares, os quais podem interferir na absorção de água e nutrientes, consequentemente, prejudicar o crescimento das mudas. Entretanto Kratz et al. (2013b), ao avaliarem a qualidade de mudas de Eucalyptus benthamii, constataram que a CE não teve influência no crescimento dos parâmetros morfológicos, semelhantes com os achados neste estudo.

Para a avaliação das propriedades físicas, os dados foram equiparados de acordo com a classificação descrita por Gonçalves e Poggiani (1996), em que os autores descreveram valores para interpretação dos resultados dessas características. Para densidade úmida, as formulações dos tratamentos contendo proporções de casca de café compostada apresentaram valores superiores, quando comparadas com os formulados com casca de arroz compostada. O tratamento T5, composto por $20 \%$ de fibra de coco, $20 \%$ de substrato comercial e $60 \%$ de casca de café compostada 
apresentou o maior valor para densidade úmida $\left(637,13 \mathrm{~kg} \mathrm{~m}^{-3}\right)$. Provavelmente ocorreu devido à maior porcentagem da casca de café contida nessa formulação. Esse valor observado pode ser considerado como aspecto negativo para o crescimento das mudas, pois o excesso de umidade pode afetar as raízes das mudas,pela redução da oxigenação no substrato, aumento da presença patógenos e possível podridão radicular.

\section{Tabela 2 - Caracterização física e química dos substratos formulados para produção de mudas de Eucalyptus urophylla, Peltophorum dubium e Eremanthus erythropappus}

Table 2 - Physical and chemical characterization of the substrates formulated for the production of Eucalyptus urophylla, Peltophorum dubium and Eremanthus erythropappus seedlings

\begin{tabular}{|c|c|c|c|c|c|c|c|c|}
\hline \multirow{2}{*}{ Trat. } & \multirow{2}{*}{ Proporções } & $\mathrm{pH}$ & $\mathrm{CE}$ & DU & DS & PT & EA & CRA (50) \\
\hline & & H2O & $\mathrm{mS} \mathrm{cm}^{-1}$ & $\mathrm{~kg} \mathrm{~m}^{-3}$ & $\mathrm{~kg} \mathrm{~m}^{-3}$ & $\%$ & $\%$ & $\%$ \\
\hline T1 & $20 \mathrm{FC}-20 \mathrm{SC}-60 \mathrm{COA}-0 \mathrm{COC}$ & 4,79 & 1,22 & 476,37 & 264,22 & 85,84 & 43,75 & 29,35 \\
\hline $\mathbf{T} 2$ & $20 \mathrm{FC}-40 \mathrm{SC}-40 \mathrm{COA}-0 \mathrm{COC}$ & 5,67 & 1,16 & 448,38 & 294,29 & 82,79 & 34,78 & 33,21 \\
\hline T3 & $20 \mathrm{FC}-60 \mathrm{SC}-20 \mathrm{COA}-0 \mathrm{COC}$ & 4,89 & 1,06 & 533,94 & 272,02 & 86,28 & 37,13 & 35,63 \\
\hline T4 & $20 \mathrm{FC}-20 \mathrm{SC}-30 \mathrm{COA}-30 \mathrm{COC}$ & 5,56 & 1,23 & 461,49 & 292,79 & 84,58 & 46,21 & 31,16 \\
\hline T5 & $20 \mathrm{FC}-20 \mathrm{SC}-0 \mathrm{COA}-60 \mathrm{COC}$ & 5,41 & 1,65 & 637,13 & 308,17 & 87,72 & 38,58 & 38,59 \\
\hline T6 & $20 \mathrm{FC}-40 \mathrm{SC}-0 \mathrm{COA}-40 \mathrm{COC}$ & 4,98 & 1,54 & 627,46 & 273,82 & 87,38 & 41,84 & 39,10 \\
\hline T7 & $20 \mathrm{FC}-60 \mathrm{SC}-0 \mathrm{COA}-20 \mathrm{COC}$ & 4,80 & 1,25 & 579,80 & 275,70 & 86,27 & 41,27 & 37,51 \\
\hline T8 & $20 \mathrm{FC}-80 \mathrm{SC}-0 \mathrm{COA}-0 \mathrm{COC}$ & 4,46 & 1,01 & 619,54 & 252,93 & 88,63 & 38,05 & 40,11 \\
\hline
\end{tabular}

Fonte: Autores (2019)

Em que: Trat. = tratamentos; $\mathrm{pH}=$ potencial hidrogeniônico determinado em água com diluição 1:5 (v/v);CE = condutividade elétrica obtida em solução 1:5 (v/v); DU = densidade úmida; DS = densidade seca; $\mathrm{PT}$ = porosidade total; EA = espaço de aeração; CRA50 = capacidade de retenção de água sob sucção de $50 \mathrm{~cm}$ de coluna de água.

Quando verificado os valores para densidade seca, de forma geral, houve baixa variação para essa variável, entre as diferentes formulações dos substratos, em que todos os tratamentos apresentaram valores classificados como médio com valores situados entre 264,22 a $308,17 \mathrm{~kg}$ $\mathrm{m}^{3}{ }^{3}$ (GONÇALVES; POGGIANI, 1996).

A densidade é um parâmetro importante na avaliação da qualidade do substrato e está relacionada com outras variáveis como a porosidade. Formulações que geram substratos mais densos tendem a apresentar menor espaço entre as partículas, o que dificulta as trocas gasosas e a circulação de água, oferecendo maior resistência ao crescimento radicular. Em contrapartida, menores valores de densidade caracterizam substratos leves, que podem proporcionar menor capacidade de suporte para as plantas, e menor capacidade de retenção de água (GONÇALVES; POGGIANI, 1996). Por esse motivo, é indicado o uso de constituintes em proporções adequadas (FARIA et al., 2016; SOUSA et al., 2016), para que não haja impedimento no crescimento das raízes.

$\mathrm{Na}$ avaliação da porosidade, foi verificado que apenas os tratamentos $\mathrm{T} 2 \mathrm{e} \mathrm{T} 4$, com valores de $82,79 \%$ e $84,58 \%$, respectivamente, foram classificados como adequados para porosidade. Os demais tratamentos variaram de 85,84 a $88,63 \%$, ficando acima dos valores considerados apropriados (GONÇALVES; POGGIANI, 1996). Observou-se que os tratamentos T5,composto por $20 \%$ de fibra de coco, $20 \%$ de substrato comercial e $60 \%$ de casca de café compostada, T6, com proporções de $20 \%$ de fibra de coco, $40 \%$ de substrato comercial e $40 \%$ de casca de café compostada e o T8, com de $20 \%$ de fibra de coco e $80 \%$ de substrato comercial foram os que 
apresentaram maior valor para porosidade.

A porosidade é muito importante quando se considera o crescimento das mudas, pois, na formação de raízes, estas exigem alto fornecimento de oxigênio e saída de gás carbônico. Nesse sentido, o substrato deve apresentar valor adequado de porosidade, permitindo assim trocas gasosas eficientes, que contribuam para o crescimento do sistema radicular e desenvolvimento da parte aérea. Com relação aos resultados para o espaço de aeração, foram encontrados valores variando entre 34,78 e 46,21\%. Em relação ao espaço de aeração, recomenda-se valores entre 20 e $40 \%$ do volume do substrato (ZORZETO et al., 2014). Desse modo, para este trabalho, as formulações dos substratos $\mathrm{T} 1, \mathrm{~T} 4, \mathrm{~T} 6$ e $\mathrm{T} 7$ não se encaixaram dentro da faixa recomendada pelos autores.

Quando avaliada a CRA a $50 \%$, a testemunha $(20 \%$ de fibra de coco e $80 \%$ de substrato comercial) apresentou o maior valor. Foi observado que as formulações contendo maiores proporções de casca de café compostada se assemelharam ao valor do tratamento testemunha, sendo superior aos valores das formulações da casca de arroz compostada.

De acordo com Wendling et al.(2007), substratos com menor capacidade de retenção de água exigem maior quantidade de irrigação, ao passo que em substratos com maior microporosidade deve haver maior controle na quantidade de irrigação utilizada, a fim de reduzir o risco de ocorrer excesso hídrico, o que é prejudicial ao crescimento das raízes das mudas. Desse modo, para as formulações contendo casca de café compostada, é necessário monitorar com mais rigor o tempo e o número de irrigações realizadas diariamente.

Após a caracterização das propriedades físicas e químicas dos substratos formulados, foram avaliados os parâmetros morfológicos e as análises qualitativas das mudas, conforme a Tabela 3. Foram encontradas diferenças estatísticas para a maioria dos caracteres avaliados. $\mathrm{Na}$ avaliação do diâmetro do coleto para Eucalyptus urophylla, não houve diferença estatística entre os tratamentos. Para Peltophorum dubium, as maiores médias em diâmetro foram obtidas nos tratamentos T2, T5, T6, T7 e T8, diferindo estaticamente dos demais. O crescimento em diâmetro das mudas de Eremanthus erythropappus variou de 3,11 a $4,20 \mathrm{~mm}$, sendo as maiores médias obtidas nos tratamentos T2, T5, T7 e T8, diferindo estaticamente dos demais.

Quando o objetivo é avaliar a qualidade das mudas em viveiro, o diâmetro do coleto é uma das características mais observadas, pois este pode indicar a capacidade de sobrevivência e, consequentemente, o crescimento das mudas em campo (GROSSNICKLE et al., 2018), apresentando ainda a vantagem da sua fácil mensuração, não necessitando de análises destrutivas para sua obtenção.

Davide et al. (2015) sugerem, como um padrão mínimo, o valor de $3 \mathrm{~mm}$ de diâmetro para espécies florestais nativas como adequado para as mudas serem expedidas para o campo. Nesse sentido, para o presente trabalho, todos os tratamentos das espécies Peltophorum dubium e Eremanthus erythropappus apresentaram valor superior ao estipulado por esses autores. Para mudas de espécies exóticas, como o Eucalyptus urophylla, indica-se um valor igual ou superior a 2 $\mathrm{mm}$ como sendo o ideal. Neste experimento, apenas o tratamento T3 não apresentou valor médio superior a $2 \mathrm{~mm}$.

Ao avaliar o crescimento inicial de mudas de Eucalyptus benthamii em substratos formulados com fibra de coco, casca de arroz carbonizada, lodo de esgoto e casca de pinus semidecomposta, Kratz et al. (2013b) observaram valores médios de diâmetro de coleto de 1,70 mm, inferiores ao encontrados para o Eucalyptus urophylla neste estudo. Dessa forma, pode-se dizer que os constituintes utilizados neste trabalho contribuíram positivamente para o crescimento das mudas com relação a essa variável, onde foi observado que as formulações com maiores proporções de casca de café favoreceram o crescimento em diâmetro, possivelmente devido às melhorias nas condições físico-hídricas do substrato.

A altura das mudas apresentou diferença significativa para o Eucalyptus urophylla, sendo os menores resultados obtidos nos tratamentos T3 e T8. Por outro lado, em mudas de Peltophorum dubium, não houve diferença estatística entre os tratamentos. Para as mudas de Eremanthus 
erythropappus, as menores médias foram verificadas nos tratamentos T1, T3, T4 e T8.

A altura das plantas é outra variável bastante utilizada na avaliação de qualidade das mudas e, assim como o diâmetro, é de fácil mensuração. Em trabalho desenvolvido por Caldeira et al. (2013), avaliando a influência de substratos alternativos na produção de mudas de Chamaecrista desvauxii, os autores observaram que as mudas apresentaram bom crescimento em altura, quando empregados os constituintes cascas de arroz e casca de café na formulação dos substratos.

Tabela 3 - Valores médios do diâmetro do coleto $(\mathrm{DC})$, altura $(\mathrm{H})$, peso da matéria seca de parte aérea (MSPA), peso da matéria seca radicular (MSR), índice de qualidade de Dickson (IQD), facilidade de retirada das mudas dos tubetes (FRT) e índice de agregação (AGR) em mudas de Eucalyptus urophylla, Peltophorum dubium e Eremanthus erythropappus, aos 120 dias após semeadura

Table 3 - Average values of the diameter of the collection (DC), height (H), weight of shoot dry matter (MSPA), weight of root dry matter (MSR), Dickson's quality index (IQD), ease of removal of the seedlings of the tubes (FRT) and aggregation index (AGR) in seedlings of Eucalyptus urophylla, Peltophorum dubium and Eremanthus erythropappus, 120 days after sowing

\begin{tabular}{|c|c|c|c|c|c|c|c|c|}
\hline \multicolumn{9}{|c|}{ Eucalyptus urophylla (eucalipto) } \\
\hline Trat. & Proporções (\%) & DC & $\mathbf{H}$ & MSPA & MSR & IQD & FRT & AGR \\
\hline--- & --------------- & $(\mathbf{m m})$ & $(\mathbf{c m})$ & (g) & $(\mathrm{g})$ & --- & --- & --- \\
\hline T1 & $20 \mathrm{FC}-20 \mathrm{SC}-60 \mathrm{COA}-0 \mathrm{COC}$ & $2,18 \mathrm{a}$ & $17,80 \mathrm{a}$ & $1,24 \mathrm{abc}$ & $0,61 \mathrm{a}$ & $0,18 \mathrm{bc}$ & $8,80 \mathrm{a}$ & $6,47 \mathrm{ab}$ \\
\hline $\mathbf{T} 2$ & $20 \mathrm{FC}-40 \mathrm{SC}-40 \mathrm{COA}-0 \mathrm{COC}$ & $2,24 \mathrm{a}$ & 18,17 a & $1,29 \mathrm{abc}$ & $0,71 \mathrm{a}$ & $0,20 \mathrm{abc}$ & $9,07 \mathrm{a}$ & $8,20 \mathrm{a}$ \\
\hline T3 & $20 \mathrm{FC}-60 \mathrm{SC}-20 \mathrm{COA}-0 \mathrm{COC}$ & $1,92 \mathrm{a}$ & $16,14 \mathrm{~b}$ & $0,84 \mathrm{c}$ & $0,52 \mathrm{a}$ & $0,13 \mathrm{c}$ & $8,73 \mathrm{a}$ & $7,80 \mathrm{ab}$ \\
\hline T4 & $20 \mathrm{FC}-20 \mathrm{SC}-30 \mathrm{COA}-30 \mathrm{COC}$ & $2,14 \mathrm{a}$ & $17,69 \mathrm{a}$ & $1,39 \mathrm{ab}$ & $0,79 \mathrm{a}$ & $0,22 \mathrm{ab}$ & $6,73 \mathrm{ab}$ & $7,80 \mathrm{a}$ \\
\hline T5 & $20 \mathrm{FC}-20 \mathrm{SC}-0 \mathrm{COA}-60 \mathrm{COC}$ & $2,22 \mathrm{a}$ & $18,38 \mathrm{a}$ & $1,29 \mathrm{abc}$ & $0,65 \mathrm{a}$ & $0,19 \mathrm{abc}$ & $7,93 \mathrm{ab}$ & $8,20 \mathrm{a}$ \\
\hline T6 & $20 \mathrm{FC}-40 \mathrm{SC}-0 \mathrm{COA}-40 \mathrm{COC}$ & $2,39 \mathrm{a}$ & $18,63 \mathrm{a}$ & $1,56 \mathrm{a}$ & $0,95 \mathrm{a}$ & $0,26 \mathrm{a}$ & $6,53 \mathrm{ab}$ & $8,47 \mathrm{a}$ \\
\hline T7 & $20 \mathrm{FC}-60 \mathrm{SC}-0 \mathrm{COA}-20 \mathrm{COC}$ & $2,10 \mathrm{a}$ & $18,63 \mathrm{a}$ & $1,26 \mathrm{abc}$ & $0,65 \mathrm{a}$ & $0,17 \mathrm{bc}$ & $7,40 \mathrm{ab}$ & $8,20 \mathrm{a}$ \\
\hline T8 & $20 \mathrm{FC}-80 \mathrm{SC}-0 \mathrm{COA}-0 \mathrm{COC}$ & $2,06 \mathrm{a}$ & $15,93 \mathrm{~b}$ & $1,00 \mathrm{bc}$ & $0,51 \mathrm{a}$ & $0,15 \mathrm{bc}$ & $5,87 \mathrm{~b}$ & $5,60 \mathrm{~b}$ \\
\hline $\mathrm{CV} \%$ & & 11,65 & 8,98 & 18,89 & 32,32 & 21,59 & 16,77 & 14,26 \\
\hline \multicolumn{9}{|c|}{ Peltophorum dubium (angico amarelo) } \\
\hline Trat. & Proporções (\%) & DC & $\mathbf{H}$ & MSPA & MSR & IQD & FRT & AGR \\
\hline--- & ------------- & $(\mathbf{m m})$ & $(\mathbf{c m})$ & $(\mathrm{g})$ & (g) & --- & --- & --- \\
\hline T1 & $20 \mathrm{FC}-20 \mathrm{SC}-60 \mathrm{COA}-0 \mathrm{COC}$ & $4,14 \mathrm{~b}$ & $13,54 \mathrm{a}$ & $1,02 \mathrm{abc}$ & $0,52 \mathrm{ab}$ & 0,37 a & $8,20 \mathrm{a}$ & $6,20 \mathrm{a}$ \\
\hline T2 & $20 \mathrm{FC}-40 \mathrm{SC}-40 \mathrm{COA}-0 \mathrm{COC}$ & $5,37 \mathrm{a}$ & $17,61 \mathrm{a}$ & $1,01 \mathrm{abc}$ & $0,45 \mathrm{ab}$ & 0,33 a & $9,54 \mathrm{a}$ & $6,73 \mathrm{a}$ \\
\hline T3 & $20 \mathrm{FC}-60 \mathrm{SC}-20 \mathrm{COA}-0 \mathrm{COC}$ & $3,95 \mathrm{~b}$ & $12,66 \mathrm{a}$ & $0,88 \mathrm{bc}$ & $0,43 \mathrm{~b}$ & $0,31 \mathrm{a}$ & 8,87 a & $4,73 \mathrm{~b}$ \\
\hline T4 & $20 \mathrm{FC}-20 \mathrm{SC}-30 \mathrm{COA}-30 \mathrm{COC}$ & $4,17 \mathrm{~b}$ & $13,68 \mathrm{a}$ & $0,98 \mathrm{abc}$ & $0,49 \mathrm{ab}$ & 0,35 a & $8,40 \mathrm{a}$ & $6,73 \mathrm{a}$ \\
\hline T5 & $20 \mathrm{FC}-20 \mathrm{SC}-0 \mathrm{COA}-60 \mathrm{COC}$ & $5,42 \mathrm{a}$ & $18,47 \mathrm{a}$ & $0,79 \mathrm{c}$ & $0,51 \mathrm{ab}$ & 0,33 a & $9,40 \mathrm{a}$ & 6,07 a \\
\hline T6 & $20 \mathrm{FC}-40 \mathrm{SC}-0 \mathrm{COA}-40 \mathrm{COC}$ & $5,22 \mathrm{a}$ & $17,34 \mathrm{a}$ & $1,35 \mathrm{a}$ & $0,55 \mathrm{ab}$ & $0,34 \mathrm{a}$ & $8,54 \mathrm{a}$ & $5,93 \mathrm{a}$ \\
\hline T7 & $20 \mathrm{FC}-60 \mathrm{SC}-0 \mathrm{COA}-20 \mathrm{COC}$ & $5,21 \mathrm{a}$ & $18,09 \mathrm{a}$ & $1,29 \mathrm{ab}$ & $0,62 \mathrm{a}$ & $0,35 \mathrm{a}$ & $9,00 \mathrm{a}$ & $4,13 \mathrm{a}$ \\
\hline T8 & $20 \mathrm{FC}-80 \mathrm{SC}-0 \mathrm{COA}-0 \mathrm{COC}$ & $5,53 \mathrm{a}$ & $17,91 \mathrm{a}$ & $1,30 \mathrm{ab}$ & $0,55 \mathrm{ab}$ & $0,33 \mathrm{a}$ & $7,60 \mathrm{a}$ & $5,20 \mathrm{a}$ \\
\hline CV\% & -------------- & 4,76 & 22,01 & 20,38 & 16,85 & 19,07 & 11,77 & 22,58 \\
\hline
\end{tabular}


Tabela 3 - Conclusão ...

Table 3 - Conclusion ...

\begin{tabular}{|c|c|c|c|c|c|c|c|c|}
\hline \multicolumn{9}{|c|}{ Eremanthus erythropappus (candeia) } \\
\hline Trat. & Proporções (\%) & DC & $\mathbf{H}$ & MSPA & MSR & IQD & FRT & AGR \\
\hline--- & -------------- & $(\mathbf{m m})$ & $(\mathbf{c m})$ & $(\mathrm{g})$ & (g) & --- & --- & --- \\
\hline T1 & $20 \mathrm{FC}-20 \mathrm{SC}-60 \mathrm{COA}-0 \mathrm{COC}$ & $3,11 \mathrm{~b}$ & $\begin{array}{c}16,97 \\
b c\end{array}$ & $2,40 \mathrm{ab}$ & $1,60 \mathrm{a}$ & $0,57 \mathrm{a}$ & $2,60 \mathrm{~cd}$ & $5,20 \mathrm{~b}$ \\
\hline T2 & $20 \mathrm{FC}-40 \mathrm{SC}-40 \mathrm{COA}-0 \mathrm{COC}$ & $4,20 \mathrm{a}$ & $25,23 \mathrm{a}$ & $3,00 \mathrm{a}$ & $1,79 \mathrm{a}$ & $0,62 \mathrm{a}$ & $2,47 \mathrm{~d}$ & $4,13 \mathrm{~cd}$ \\
\hline T3 & $20 \mathrm{FC}-60 \mathrm{SC}-20 \mathrm{COA}-0 \mathrm{COC}$ & $3,23 \mathrm{~b}$ & $\begin{array}{c}17,23 \\
\text { bc }\end{array}$ & $1,93 \mathrm{~b}$ & $2,37 \mathrm{a}$ & $0,70 \mathrm{a}$ & $3,93 \mathrm{~b}$ & $6,20 \mathrm{a}$ \\
\hline T4 & $20 \mathrm{FC}-20 \mathrm{SC}-30 \mathrm{COA}-30 \mathrm{COC}$ & $3,20 \mathrm{~b}$ & $17,13 \mathrm{~b}$ & $1,76 \mathrm{~b}$ & $1,26 \mathrm{a}$ & $0,45 \mathrm{a}$ & $3,92 \mathrm{~b}$ & $3,46 \mathrm{de}$ \\
\hline T5 & $20 \mathrm{FC}-20 \mathrm{SC}-0 \mathrm{COA}-60 \mathrm{COC}$ & 3,97 a & $22,19 \mathrm{a}$ & $2,37 \mathrm{ab}$ & $1,57 \mathrm{a}$ & $0,55 \mathrm{a}$ & $3,80 \mathrm{bc}$ & $4,67 \mathrm{bc}$ \\
\hline T6 & $20 \mathrm{FC}-40 \mathrm{SC}-0 \mathrm{COA}-40 \mathrm{COC}$ & $3,28 \mathrm{~b}$ & $23,22 \mathrm{a}$ & $1,82 \mathrm{~b}$ & $2,73 \mathrm{a}$ & $0,61 \mathrm{a}$ & $1,74 \mathrm{~d}$ & $3,47 \mathrm{de}$ \\
\hline T7 & $20 \mathrm{FC}-60 \mathrm{SC}-0 \mathrm{COA}-20 \mathrm{COC}$ & $3,99 \mathrm{a}$ & 21,90 a & $2,53 \mathrm{ab}$ & $2,84 \mathrm{a}$ & $0,86 \mathrm{a}$ & $4,33 \mathrm{~b}$ & $6,26 \mathrm{a}$ \\
\hline T8 & $20 \mathrm{FC}-80 \mathrm{SC}-0 \mathrm{COA}-0 \mathrm{COC}$ & $4,05 \mathrm{a}$ & $16,60 \mathrm{~b}$ & $1,84 \mathrm{~b}$ & $1,17 \mathrm{a}$ & $0,53 \mathrm{a}$ & $5,73 \mathrm{a}$ & $3,07 \mathrm{e}$ \\
\hline $\mathrm{CV} \%$ & -------------- & 5,4 & 12,7 & 19,06 & 47,92 & 36,14 & 17,8 & 7,5 \\
\hline
\end{tabular}

Fonte: Autores (2019)

Em que: Médias seguidas da mesma letra, na coluna e para a mesma espécie, não diferem estatisticamente entre si pelo teste Tukey, a 5\% de probabilidade de erro.

Considerando os dados observados para altura das mudas de Eucalyptus urophylla e Eremanthus erythropappus deste estudo, é possível verificar que as menores médias foram obtidas nas formulações com maior proporção de substrato comercial, como nos tratamentos T3 e T8. Desse modo, as proporções com maior porcentagem de casca de café contribuíram com o crescimento em altura dessas duas espécies, possivelmente devido ao aumento da porosidade no substrato, proporcionado por este constituinte. De maneira geral, verifica-se que os substratos que possuem a casca de café como constituinte, juntamente com o tratamento $2(20 \%$ de fibra de coco, $40 \%$ de substrato comercial e $40 \%$ de casca de arroz carbonizada), possibilitaram maior crescimento e qualidade às mudas.

Quando avaliado o peso da matéria seca da parte aérea (MSPA), em mudas do Eucalyptus urophylla e Peltophorum dubium, as maiores médias foram obtidas no T6, enquanto para as mudas de Eremanthus erythropappus o maior valor $(3,00)$ de MSPA foi observado no T2. Para o peso da matéria seca radicular (MSR), não houve diferença significativa entre os tratamentos para Eucalyptus urophylla e Eremanthus erythropappus, enquanto para Peltophorum dubium as maiores médias foram encontradas no T6. De forma geral, para essas duas variáveis, o comportamento entre as espécies foi diferente, mas é possível sugerir como pior substrato, para todas as espécies, o T3, seguido do T8 (para duas espécies). Em contrapartida, pode-se sugerir como melhores substratos, o T1, T2 e T7, para todas as espécies, além do T6, que apesar de não ter apresentado os maiores valores de MSPA para Eremanthus erythropappus, foi um dos melhores para MSR dessa espécie e para ambas as características de Eucalyptus urophylla e Peltophorum dubium.

$\mathrm{Na}$ avaliação do IQD nas mudas do Eucalyptus urophylla, o maior valor foi obtido no tratamento $\mathrm{T} 6$, com proporções de $20 \%$ de fibra de coco, $40 \%$ de substrato comercial e $40 \%$ de casca de café compostada, evidenciando que essa formulação foi essencial para o crescimento das mudas dessa espécie. Para as outras duas espécies, não houve diferença significativa entre os tratamentos. O índice de qualidade de Dickson (IQD) é muito utilizado na avaliação de qualidade de mudas, tendo como vantagens a análise conjunta dos demais parâmetros morfológicos 
em uma única avaliação. Este parâmetro é muito empregado nas avaliações, pois considera a robustez e o equilíbrio entre as características avaliadas e, quanto maior seu valor, maior é o grau de qualidade da muda dentro de um mesmo lote (AGUIAR et al., 2011).

Como análise qualitativa, a avaliação de retirada das mudas dos tubetes (FRT) é importante, pois está relacionada com a qualidade do torrão formado e considera se esta é uma atividade onerosa ou não (WENDLING et al., 2007). Quanto mais fácil for essa operação, menor será o tempo destinado ao preparo das mudas para o plantio. Porém, a FRT pode estar relacionada com o baixo enraizamento, apresentando fácil operação, mas como aspecto negativo, baixa quantidade de biomassa radicial, sendo prejudicial para o desenvolvimento da planta em campo (FRAGOSO et al., 2016).

$\mathrm{Na}$ avaliação da FRT para o Eucalyptus urophylla, o menor valor foi observado no tratamento testemunha. Para Peltophorum dubium, não houve diferenças significativas entre os tratamentos. Para Eremanthus erythropappus, os resultados encontrados para todas as formulações testadas foram relativamente baixos. A maior média para essa variável foi obtida em mudas do T8, sendo esse tratamento estaticamente superior aos demais

Avaliando substratos alternativos à base de casca de arroz carbonizada, combinada com fibra de coco, para Eucalyptus camaldulensis, Kratz et al. (2016) observaram médias de facilidade de retirada da muda do tubete próximas a 8. Para mudas de Eucalyptus urophylla, neste trabalho, as mudas dos tratamentos T1, T2 e T3 apresentaram média acima de 8, corroborando com dados observados por Stuepp et al. (2016), na avaliação da FRT em mudas de Piptocarpha angustifolia.

Em algumas espécies, a dificuldade de retirada da muda do tubete está relacionada com a maior quantidade de raízes, ocasionando maior compactação, o que dificulta a liberação do torrão. A espécie Eremanthus erythropappus apresentou elevada quantidade de raízes, que dificultou essa operação. Porém, o fato de maior quantidade de raízes atrapalhar a FRT, pode indicar que esses tratamentos apresentaram maior formação de raízes, sendo considerado essencial para o melhor crescimento das mudas em campo. De acordo com Melo et al. (2018), a maior quantidade de raízes pode indicar melhor agregação do substrato à muda e posterior maior índice de sobrevivência das mudas, quando plantadano campo.

Quando avaliada a agregação das mudas ao substrato (AGR), em mudas de Eucalyptus urophylla, o menor valor foi observado no tratamento testemunha, semelhante ao observado para FRT. Para a espécie Peltophorum dubium, não houve diferença estatística entre tratamentos. Na avaliação das mudas de Eremanthus erythropappus, os valores encontrados foram considerados baixos (WENDLING et al., 2007), em que as maiores médias alcançadas foram nos tratamentos T3 e T7, diferindo estaticamente dos demais. Tal fato ocorreu, pois a avaliação da AGR é posterior a da FRT; além disso, como a FRT foi influenciada pela alta presença de raízes, a avaliação da AGR foi comprometida.

Quanto mais vigoroso o enraizamento, mais coeso e mais agregado é o substrato, devido ao maior desenvolvimento de raízes laterais. O substrato, para ser considerado adequado para a produção de mudas, deve fornecer condições adequadas ao crescimento radicular e permitir boa agregação das raízes (MELO et al., 2018), para que o torrão não se rompa quando for removido do tubete, o que pode ocasionar exposição das raízes ao ressecamento e dificultar a sobrevivência das mudas em campo (WENDLING et al., 2007). Tal fato foi observado nas mudas de Eremanthus erythropappus, na qual a espécie teve crescimento elevado de raízes, o que dificultou as operações de manejo da muda no viveiro. No entanto, como aspecto positivo, deve-se ressaltar que a quantidade de biomassa radicular pode indicar maior capacidade de sobrevivência da muda nas condições adversas em campo.

Quando a finalidade é a avaliação da produção de mudas de qualidade, a facilidade de retirada da muda do tubete e agregação das raízes ao substrato tornam-se características importantes. Como na literatura científica são encontrados poucos estudos com essas variáveis qualitativas, indicam-se essas avaliações para um número maior de espécies, sempre com o objetivo de produção de mudas de alta qualidade e com o menor custo operacional, que tenham 
maior capacidade de sobrevivência em campo e possam ser empregadas na formação de florestas produtivas ou para fins de restauração.

Para melhor compreensão dos parâmetros avaliados em relação aos tratamentos formulados, por meio da análise dos componentes principais (PCA) foi possível agrupar os tratamentos, conforme seu desempenho em relação às variáveis avaliadas, como pode ser visto na Figura 1.

Figura 1 - Projeção dos componentes principais mostrando em detalhes o desempenho dos oito tratamentos em relação às características avaliadas, no crescimento de mudas de Eucalyptus urophylla (A), Peltophorum dubium (B) e Eremanthus erythropappus (C), respectivamente

Figure 1 - Projection of the main components showing in detail the performance of the eight treatments in relation to the evaluated characteristics, in the growth of seedlings of Eucalyptus urophylla (A), Peltophorum dubium (B), and Eremanthus erythropappus (C), respectively

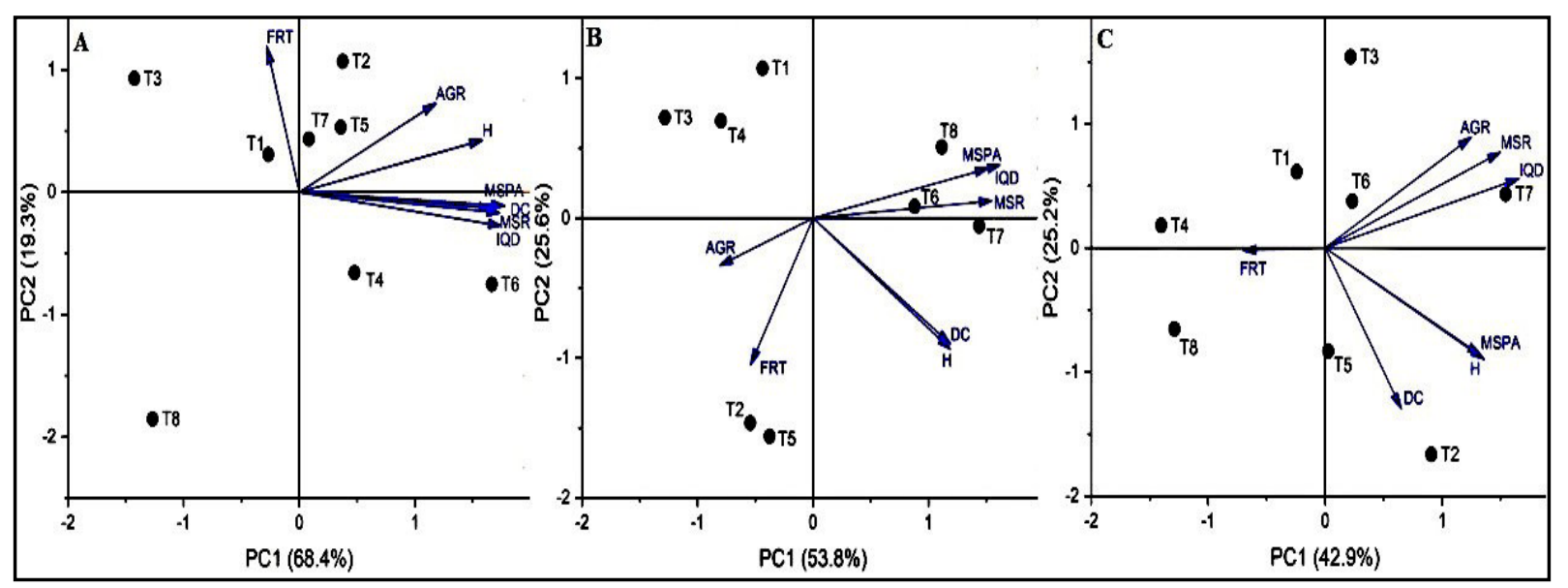

Fonte: Autores (2019)

Na Figura 1 é possível observar a interação entre os componentes para as três espécies do presente estudo. Na PCA biplot de PC1 e PC2, pela matriz de correlação dos resultados das propriedades médias, resultou em uma variabilidade total de $87,7 \%, 79,4 \%$ e $68,1 \%$ para as variáveis avaliadas de Eucalyptus urophylla, Peltophorum dubium e Eremanthus erythropappus respectivamente (Figura 1). Para cada eixo, os maiores valores indicam a variável mais representativa, explicando dessa forma a maior parcela da variância do conjunto original de dados (CRUZ et al., 2018).

Para o Eucalyptus urophylla, ao analisar os dois componentes principais, nota-se uma nítida separação entre os tratamentos. Observa-se que os tratamentos T3 e T8 não se correlacionaram com a variáveis analisadas, sendo estes os tratamentos que apresentaram as menores médias para a maioria dos parâmetros estudados, conforme discutido anteriormente. Em contrapartida, os tratamentos T5 e T6 apresentaram as maiores correlações para as variáveis DC, H, IQD, MSR, MSPA e AGR.

Para a espécie Peltophorum dubium, nota-se que os tratamentos T1, T3 e T4 não se correlacionaram com nenhuma das variáveis analisadas, comprovando assim que esses tratamentos apresentaram pior crescimento das mudas, não sendo indicados para a produção de mudas dessa espécie. Em contrapartida, os tratamentos T6, T7 e T8 apresentaram alta correlação com as características MSR, MSPA e IQD, demonstrando resultados positivos na avaliação de qualidade de mudas dessa espécie.

Ao analisar o agrupamento dos tratamentos para a Eremanthus erythropappus, percebe-se que houve maior correlação nos tratamentos T6 e T7, com as variáveis AGR, MSR e IQD. O T2 
apresentou forte correlação como DC e correlação moderada com H e MSPA.

A análise de componentes principais foi considerada apropriada, pois demonstrou graficamente de forma eficiente, o comportamento dos diferentes tratamentos em relação às variáveis avaliadas, sendo uma ferramenta importante quando se considera a acurácia de um conjunto de dados avaliados (CRUZ et al., 2018; LI et al., 2018).

De forma geral, as mudas apresentaram crescimento adequado, consideradas aptas para o plantio, com ótimo crescimento dos parâmetros fitométricos. A casca de café apresentou potencial para ser utilizada na formulação de substrato, pois proporcionou melhorias na qualidade das mudas produzidas. Dessa forma, esse resíduo torna-se mais uma alternativa viável a ser empregado como constituinte de substrato na produção de mudas de espécies florestais.

\section{Conclusões}

Os resultados encontrados atestaram que as mudas dos tratamentos formulados com casca de café compostada apresentaram valores superiores para a maioria das variáveis analisadas, quando comparadas com as mudas produzidas nos tratamentos formulados com casca de arroz compostada e no tratamento testemunha.

A casca de café compostada, combinada com proporções de fibra de coco e substrato comercial apresentou potencial para ser utilizada na produção de mudas das três espécies florestais. Indica-se a proporção $20 \%$ de fibra de coco, com $40 \%$ de substrato comercial e $40 \%$ de casca de café compostada, na formulação de substratos, para a produção de mudas de Eucalyptus urophylla, Peltophorum dubium e Eremanthus erythropappus.

\section{Agradecimentos}

Os autores agradecem a Cemig, à UFLA e à Coordenação de Aperfeiçoamento de Pessoal de Nível Superior (CAPES).

\section{Referências}

ABREU, A. H. M.; LELES, P. S. S.; MELO, L.M.; OlIVEIRA, R. R.; FERREIRA, D. H. A. A. Caracterização e potencial de substratos formulados com biossólido na produção de mudas de Schinus terebinthifolius Raddi. e Handroanthus heptaphyllus (Vell.) Mattos. Ciência Florestal, Santa Maria, v. 27, n. 4, p. 1179-1190, 2017.

AGUIAR, F. F. A.; KANASHIRO, A.; TAVARES, A. R.; NASCIMENTO, T. D. R.; ROCCO, F. M. Crescimento de mudas de pau-brasil (Caesalpinia echinata Lam.), submetidas a cinco níveis de sombreamento. Revista Ceres, Viçosa, v. 58, n. 6, p. 729-734, 2011.

ALVARES, C. A.; STAPE, J. L.; SENTELHAS, P. C.; GONÇALVES, J. L. M.; SPAROVEK, G. Kö ppen'sclimateclassificationmap for Brazil. Meteorologische Zeitschrift, Sturtgart, v. 22, n. 6, p. 711-728, 2013.

CALDEIRA, M.V.W.; DELARMELINA, W. M.; FARIA, J. C. T.; JUVANOL, R.S. Substratos alternativos na produção de mudas de Chamaecrista desvauxii. Revista Árvore, Viçosa, v. 37, n. 1, p. 31-39, 2013.

COMPANHIA NACIONAL DE ABASTECIMEMTO (CONAB). Acompanhamento da Safra Brasileira de Café, Safra 2018, segundo levantamento, maio/2018. Conab, 2018. Disponível em https:/www.embrapa.br/busca-de-noticias/-/noticia/34724227/producao-dos-cafes-do- 
brasilequivale- a-36-da-producao-mundial-em-2018. Acesso em: 18/05/2020.

CRUZ, D. G.; NOGUEIRA, F. C.; ROSSI, D. Q.; PEREIRA, T. M.; FERREIRA, R. F.Análise fatorial multivariada aplicada na caracterização de contaminantes de um depósito de minério de ferro. Tecnologia em Metalurgia, Materiais e Mineração, São Paulo, v. 15, n. 3, p. 248-253, 2018.

DA ROS, C. O.; REX, F. E.; RIBEIRO, I. R.; KAFER, P. S.; RODRIGUES, A. C.; SILVA, R. F.; SOMAVILLA, L. Uso de substrato compostado na produção de mudas de Eucalyptusdunnii e Cordia trichotoma. Floresta e Ambiente, Seropédica, v. 22. n. 4, p. 549-558, 2015.

DAVIDE, A. C.; BOTELHO, S. A. Fatores que afetam a qualidade de mudas destinadas aos projetos de restauração de ecossistemas florestais. In: DAVIDE, A. C.; BOTELHO, S. A. (Ed.). Fundamentos e métodos de restauração de ecossistemas florestais: 25 anos de experiência em matas ciliares. Lavras: Ed. UFLA, 2015. p. 181-274.

DICKSON, A.; LEAF, A. L.; HOSNER, J. F. Quality appraisal of white spruce and white pine seedling stock in nurseries. Forest Chronicle,[s.l.], v. 36, p.10-13, 1960.

FARIA, J. C. T.; CALDEIRA, M. V. W.; DELARMELINA, W. M.; ROCHA, R. L. F.Substratos alternativos na produção de mudas de Mimosa setosa Benth. Ciência Florestal, Santa Maria, v. 26, n. 4, p. 1075-1086, 2016.

FERREIRA, D. F. Sisvar: a guide for its bootstrap procedures in multiple comparisons. Ciência e Agrotecnologia, Lavras, v. 38, n. 2, p.109-112, 2014.

FRAGOSO, R. O.; STUEPP, C. A.; APARECIDO CARPANEZZI, A. A.; WENDLING, I.; ZUFFELLATO-RIBAS, K. C.; KOEHLER, H. S. Substratos renováveis na produção de mudas de Ficus enormis proveniente de jardim clonal. Pesquisa Florestal Brasileira, Brasília, v. 36, p. 537, 2016.

GERVASIO, C. R.; SILVA, A. C. S.; SARMENTO, M. B.; NETTO, C. G.; PINHEIRO, L. M. C.; OLIVEIRA, C. J. Substratos na produção de mudas de espécies nativas do sul do Brasil. Magistra, Crus das Almas, v. 28, n. 2, p. 268-272, 2016.

GONÇALVES, L. M.; POGGIANI, F. Substratos para produção de mudas florestais. In: Congresso latino americano de ciência do solo, 13. Águas de Lindóia,1996. Resumos. Piracicaba, Sociedade Latino Americana de Ciência do Solo, CD-ROM, 1996.

GROSSNICKLE, S. C.; MACDONALD, J. E. Seedling quality: history, application, and plant atributes. Forests,Basel, v. 9, p. 283, 2018.

KRATZ, D.; WENDLING, I. Crescimento de mudas de Eucalyptus camaldulensis em substratos à base de casca de arroz carbonizada. Revista Ceres, Viçosa, v. 63, p. 348-354, 2016.

KRATZ, D.; WENDLING, I. Produção de mudas de Eucalyptus dunnii em substratos renováveis. Floresta, Curitiba, v. 43, n. 1, p. 125-136, 2013.

KRATZ, D.; WENDLING, I.; NOGUEIRA, A. C.; SOUZA, P. V. D. Substratos renováveis na produção de mudas de Eucalyptus benthamii. Ciência Florestal, Santa Maria, v. 23, n. 4, p. 607621, 2013b.

KRAUSE, M. R.; MONACO, P. A. V.; HADDADE, I. R.; MENEGHELLI, L. A. M.; SOUZA, T. D. Aproveitamento de resíduos agrícolas na composição de substratos para produção de mudas de tomateiro. Horticultura Brasileira, Brasília, v. 35, p. 280-285, 2017.

LI, X.; YMING, F. Y.; TING, S.; JIANG, J.; LIU, Y. Correlating emulsion properties to micro encapsulation efficacy and nutrients retention in mixed proteins system. Food Research International, [s.1.], n, 115, p. 44-53, 2018.

MELO, L. A.; ABREU, A. H. M.; LELES, P. S. S.; OLIVEIRA, R. R.; SILVA, D. T. Qualidade e crescimento inicial de mudas de Mimosa caesalpiniifolia Benth. produzidas em diferentes volumes 
de recipientes. Ciência Florestal, Santa Maria, v. 28, n. 1, p. 47-55, 2018.

MELO, L. A.; PEREIRA, G. A.; MOREIRA, E. J. C.; DAVIDE, A. C.; SILVA, E. V.; TEIXEIRA, L. A. F. Crescimento de mudas de Eucalyptus grandis e Eremanthus erythropappus sob diferentes formulações de substrato. Floresta e Ambiente, Seropédica, v. 21, n. 2, p. 234-242, 2014.

Ministério da Agricultura, Pecuária E Abastecimento (MAPA). Instrução Normativa SDA no 17. Diário Oficial da União - Seção 1, no 99, 24 de maio de 2007. Métodos Analíticos Oficiais para Análise de Substratos para Plantas e Condicionadores de Solo. Brasília, 2007.

R CORE TEAM. R: A language and environment for statistical computing. Vienna: R Foundation for Statistical Computing, 2016.

SILVA, R. B. G.; SIMÓES, D.; SILVA, M. R. Qualidade de mudas clonais de Eucalyptus urophylla $x$ Eucalyptus grandis em função do substrato. Revista Brasileira de Engenharia Agrícola e Ambiental, Campina Grande, v. 16, n. 3, 2012.

SOUSA, H. S.; SILVA, H. S.; GONÇALVES, D. S.; SOUZA, P. A.; SANTOS, A. F. Efeito de diferentes sistemas de produção de mudas e substratos no desenvolvimento de Enterolobium Contortisiliquum. Revista da Universidade Vale do Rio Verde, Três Corações, v. 14, n. 2, p. 10931100, 2016.

STUEPP, C. A. WENDLING, I.; KOEHLER, H. S.; ZUFFELLATO-RIBAS, K. C. Quality of clonal plants of Piptocarpha angustifolia in different renewable substrates and seasons of the year. Pesquisa Agropecuária Brasileira, Brasília, v. 51, p. 1821-1829, 2016.

VALERI, S. V.; CORRADINI, L. Fertilização em viveiro para produção de mudas de Eucalyptus e Pinus. In: GONÇALVES, J. L. M.; BENEDETTI, V. Nutrição e fertilização florestal. Piracicaba: IPEF, 2000. p. 168-190.

WENDLING, I.; GUASTALA, D; DEDECEK, R. Características físicas e químicas de substratos para produção de mudas de Ilex paraguariensis St. Hil. Revista Árvore, Viçosa, v. 31, p. 209-220, 2007.

ZORZETO, T. Q.; DECHEN, S. C. F.; ABREU, F. F. J. Caracterização física de substratos para plantas. Bragantia, Campinas, v. 73, n. 3, p. 300-311, 2014. 